\title{
Various Types of Advanced Technologies in Sports
}

\author{
Dr. Prof. T.F Gulhane \\ Rajiv Gandhi College of Engineering Research \& Technology, Chandrapur, Maharashtra, India.
}

\begin{abstract}
Technological advancement is a natural process, and with its introduction into a sport, athletes simply become "better". Technologies in sports are man-made means developed to reach human interests or goals in or relating to a particular sport. Technology in sports is a technical means by which athletes attempt to improve their training and competitive surroundings in order to enhance their overall athletic performance. It is the knowledge and application of using specialized equipment and the latest modern technologies to perform tasks more efficiently. In this, paper discussed efficient technologies that will help in enhancing the performance and quality of sports.
\end{abstract}

Keywords: Technological advances, Athletes consideration, High speed cameras

\section{Introduction}

Many equipment, such as monocoque racing bicycle race and buoyant swimsuits, this same effect was reverse by rule soon after the changes where introduce bike design returned to the conventional divert frames, and swimsuits reverted to less buoyant, and more permeable materials. Yet independent of whether, or not rukes are imposed on technological advances, sport technology is almost always about the same principle: energy.

Sport performance is define by the energy produce by the athlete and released into the environment. The energy must be exclusively produce by the athlete, and must not come from else (example. An external energy source.) However, not all off the energy produce the athlete is necessarily released into the environment. Common source energy loss (non-conservative, non-recoverable energy) is: external fiction (example sliding friction in skiing or rolling friction in cycling); internal friction; aerodynamic drag; hydrodynamic sound; heat; vibrations; and the energy required for stability etc.

The task of the sports engineer is finding to energy leaks and develops ways to mend them. Solutions are readily at hand. For example, sport engineer can develops swimsuits that left the body slightly out of the water, thereby reducing water resistance and increasing air drag or perform wind turner test of skiers to find way to optimize the tucked racing position.

\section{Benefits Of Advanced Sporting Technologies}

Recent development in sporting technologies has created a variety of products aimed at improving and increasing athletic performance. Athletic health can be modern sporting technologies such as heart rate, monitors, pedometers and body- fat monitors, through this a, greater depended knowledge of the human body and its potential has been recognized, allowing athletes to train complete in sports to much older age. Participant safety at all times has also been made possible through the development of certain sporting equipment, such as helmets and body protection which are used in boxing and ice hockey to help prevent injuries. Modern sporting technologies have also made competition judging easier and more accurate, and spectator interest and excitement is enhanced by broadcasting and in-stadium displays (scoreboard).

\section{How Can Technology Are Used To Enhance Athletic Performance}

Sports gear such as clothing and footwear should be user-friendly and include valuable properties such as strength, flexibility, density, thickness, durability, toughness, resistance to moisture and more importantly cost. Footwear is generally considered more for comfort and injury avoidance rather than performance enhancement. Whereas clothing such as the full body suits used in swimming. Are often claimed to rationalize the competitor's performance times where winning or losing the race is hundredths of a second. In tennis racket has been created in order to provide enhanced ball speed, and reduce the potential vibration that can lead to a condition known as tennis elbow. In other sorting equipment such as the golf club, the overall mass of the club the overall mass of the club has decreased which is believed to result in a greater achievable distance and possible a more precise shot. The bicycle has also undergone modern day advances with the development of specialist wheels, pneumatic tyres; break levers and pedals, which are all aimed at increasing stability and rigidity of the bicycle. The most efficient technology is the high speed cameras which are used in sports. There are various high speed cameras such as high speed - greater than 100 FDS (frames per second) still cameras, motion cameras. Motion cameras are more useful in sports Medicine, Physical education and sports, DV Camera-60 FPS, Digitals Still Camera-0 to 1 FPS, Multiple Cameras requires identical format and frame rate. 
High speed cameras uses in physical education and sports for an example; Shorten the learning curve, Coaching, training and Analysis, Kinematic, Biomechanics Research, locomotives and Behavior Analysis, physical Therapy, Injury prevention, In refereeing, Cricket -Third Umpire, Tennis ball, In out ,Table-players shots, Equestrian-Horse running analysis, Chest finish (spirits), Correct Technique Model presentation or Animation. Comparison of the technique (trainees) with elite to communicate correct techniques.

\section{How Can Technologies By Used To Analyze Athletic Performance:}

Technologies such as CAD (Computer aided design) can play a major role in the improvement of sporting equipment. CAD allows virtual design and testing techniques to be applied to all aspects of sport and equipment research and development. CAD offers an efficient means of considering an assessing new products and ideas and is primarily used to improve safety, comfort and effectiveness of specialized sports equipments; $\mathrm{CAD}$ is also used regularly in the justification of physical fact figures and for both competitive and training circumstances. Other technologies such as "smart" equipment can be used evaluate performance. Example of "smarts" equipments technologies include devices used for exercise stress testing and cardiovascular assessment, human reaction time and frequency of movement meters, and jump and run characteristics devices.

The use modern technology in sports may mean that competition at the uppermost level is only affordable to the leading top athletes due to the potential high costs of specialized sports equipments, in those sports incorporating individuals with a particular disability, there are a variety of methods in which assistance can be given. For example, modifications to buildings can be made to make them wheelchair accessible, specialized equipments can also be produced and training to sports members can be offered in order to give specific assistance to those with a disability.

\section{Conclusion}

Technology also makes better information available to the coach's athletes and spectators, and this serves the sport on many different levels; it enables better match analysis, performance ranking, player selection, sports statistics and predictions and in general makes the more interesting. The Hawk-Eye system is a typical example of a beneficial technology that provides a multitude of information during cricket and tennis matches.

Recent developments in sporting technologies have created a variety of products aimed at improving and increasing athletic performance.

\section{References}

[1] Bandura, A. (1986) Social foundations of thought and action: A social cognitive theory. Englewood Cliffs, NJ: Prentice Hall.

[2] Beesley, S. and Mutrie, N. (1997) Exercise is beneficial adjunctive treatment in depression. British Medical Journal, $315,1542$.

[3] Biddle, S. J. H. (1997) Current trends in sport and exercise psychology research. The Psychologist: Bulletin of the British Psychological Society, 10(2), 63-9.

[4] Brownson, R. C., D. A. Jones, M. Pratt, C. Blanton, and G.W. Heath. 2000. Measuring Physical Activity with the Behavioral Risk Factor Surveillance System. Medicine and Science in Sports and Exercise, Vol. 32, No. 11, pp. 1913-1918.

[5] Biddle, S. J. H., Fox, K. R. and Edmunds, L. (1994) Physical activity promotion in primary health care in England. London: Health Education Authority 